\title{
Helicobacter pylori Infection with Atrophic Gastritis Is an Independent Risk Factor for Advanced Colonic Neoplasm
}

Ji Young Lee ${ }^{1}$, Hye Won Park ${ }^{1}$, Ji Young Choi ${ }^{1}$, Jong-Soo Lee ${ }^{1}$, Ja Eun Koo ${ }^{1}$, Eun Ju Chung ${ }^{1}$, Hye-Sook Chang ${ }^{1}$, Jaewon Choe $^{1}$, Dong-Hoon Yang ${ }^{2}$, Seung-Jae Myung ${ }^{2}$, Hwoon-Yong Jung ${ }^{2}$, Suk-Kyun Yang ${ }^{2}$, and Jeong-Sik Byeon ${ }^{2}$

${ }^{1}$ Health Screening and Promotion Center and ${ }^{2}$ Department of Gastroenterology, Asan Medical Center, University of Ulsan College of Medicine, Seoul, Korea

\section{See editorial on page 867.}

Background/Aims: Helicobacter pylori is a major risk factor for atrophic gastritis (AG) and gastric cancer. The correlation between $H$. pylori, AG and colorectal neoplasm (CRN) has only been examined in a limited number of studies, and findings have been inconclusive. We aimed to investigate the association between $H$. pylori infection status, AG and advanced CRN. Methods: This cross-sectional study investigated the relationship between the presence of serum antiH. pylori lgG antibodies, AG, and advanced CRN in 6,351 consecutive asymptomatic subjects who underwent a screening colonoscopy. Results: A total of 316 participants (5.0\%) had advanced CRN. H. pylori seropositivity was $61.3 \%$. In a univariate analysis, the presence of $H$. pylori infection was associated with advanced CRN (odds ratio [OR], 1.49; 95\% confidence interval [Cl], 1.17 to $1.91 ; p=0.001)$. H. pylori infection was associated with an increased risk of advanced CRN after adjusting for clinically relevant confounders (OR, 1.34; $95 \% \mathrm{Cl}, 1.04$ to $1.72 ; \mathrm{p}=0.023$ ). H. pylori-related AG was significantly associated with the risk of advanced CRN (OR, 1.40; 95\% Cl, 1.03 to 1.91; $p=0.030$ ), whereas H. pylori infection without AG was not. Conclusions: $H$. pylori infection increased the risk of advanced CRN, especially when it was combined with AG. Strict colonoscopy screening and surveillance may be warranted in those with $H$. pylori-positive AG. (Gut Liver 2016;10:902-909)

Key Words: Helicobacter pylori; Gastritis; Atrophy; Neoplasms

\section{INTRODUCTION}

Helicobacter pylori was classified as a Group 1 carcinogen in human gastric carcinogenesis. ${ }^{1}$ The overall estimate of $H$. pylori seroprevalence in middle aged adults is 74\% in developing countries and 58\% in developed countries. Eastern Asia including Korea showed 55\% to 64\% prevalence of $\mathrm{H}$. pylori seropositivity. ${ }^{2}$ Besides its well-known relation to gastric adenocarcinoma, $H$. pylori has been also linked with various extragastric malignancies. One of the most interesting and so far best analyzed associations is $H$. pylori and colorectal neoplasm (CRN). ${ }^{3,4}$ It was the first recognized that CRN was associated with an increased prevalence of $H$. pylori infection in the 1990s. ${ }^{5}$ However, the association between $H$. pylori and CRN was inconsistent between studies. Although some studies demonstrated modest association between $H$. pylori and $\mathrm{CRN}^{6-9}$ others did not show the positive relationship. ${ }^{10,11}$

Gastric mucosal atrophy is a late sequela from chronic $H$. pylori infection. The long-term presence of $H$. pylori infection leads to atrophic gastritis (AG) in $8.1 \%$ of patients per year ${ }^{12}$ and is associated with a 10 -fold increased risk of AG. ${ }^{13}$ Premalignant gastric lesions such as AG or dysplasia had a significant risk of gastric cancer ${ }^{14}$ and gastric cancer and/or adenoma was associated with higher prevalence of colorectal cancer and adenoma. ${ }^{15}$ However, the correlation between H. pylori-related AG and CRN has been investigated only in a limited number of studies and it is inconclusive whether AG increases the risk of CRN. One study showed an insignificant association between colon cancer and AG diagnosed by pepsinogen I/II ratio or histology. ${ }^{11}$ In contrast, a recent study showed that intestinal metaplasia, which is usually accompanied by AG, was strongly associated with any type of CRN. ${ }^{16}$

\footnotetext{
Correspondence to: Jeong-Sik Byeon

Department of Gastroenterology, Asan Medical Center, 88 Olympic-ro 43-gil, Songpa-gu, Seoul 05505, Korea

Tel: +82-2-3010-3905, Fax: +82-2-476-0824, E-mail: jsbyeon@amc.seoul.kr

Received on July 21, 2015. Revised on November 5, 2015. Accepted on February 2, 2016. Published online July 27, 2016

pISSN 1976-2283 eISSN 2005-1212 https://doi.org/10.5009/gnl15340

Ji Young Lee and Hye Won Park contributed equally to this work as first authors.

(a) This is an Open Access article distributed under the terms of the Creative Commons Attribution Non-Commercial License (http://creativecommons.org/licenses/by-nc/4.0) which permits unrestricted non-commercial use, distribution, and reproduction in any medium, provided the original work is properly cited.
} 
Therefore, the present study was to investigate the association between $H$. pylori infection and CRN, especially, advanced CRN. Furthermore, we aimed to evaluate whether AG plays some role in the relation between $H$. pylori infection and advanced CRN.

\section{MATERIALS AND METHODS}

\section{Study design and population}

This retrospective cross-sectional study was conducted using a consecutive series of subjects who underwent screening colonoscopy and $H$. pylori serology testing as part of a health checkup program from January 2012 to November 2013 at the Health Screening and Promotion Center in Asan Medical Center, Seoul, Korea. All subjects to be screened provided informations to a standard questionnaire regarding their personal medical history including colorectal cancer, present medications, family history of colorectal cancer in first-degree relatives, and lifestyle such as smoking and alcohol consumption. Physical examinations, laboratory assays, imaging studies, and colonoscopy were performed after fasting for at least 12 hours. The examination data were recorded in a centralised electronic medical record system. Endoscopic findings and pathologic findings were recorded as coding system as well as descriptive form in electronic medical record system.

We excluded subjects who had a history of $H$. pylori eradication, a history of colorectal cancer, incomplete colonoscopy due to poor bowel preparation and cecal intubation failure, and unavailable esophagogastroduodenoscopy (EGD) data. The Institutional Review Board of the Asan Medical Center (Seoul, Korea) approved this study (IRB No. 2013-1072).

\section{Physical parameters and laboratory assays}

Waist circumference, blood pressure, triglyceride level, highdensity lipoprotein cholesterol level, fasting glucose level, and medical history were obtained to evaluate the presence of metabolic syndrome. Metabolic syndrome was defined as having at least three of the criteria set by the National Cholesterol Education Programme/Adult Treatment Panel III criteria, as updated by the American Heart Association/National Heart, Lung, and Blood Institute. ${ }^{17}$ The body mass index (BMI) was calculated as the weight in kilograms divided by the square of the height in meters. Obesity was defined as a BMI $\geq 25 \mathrm{~kg} / \mathrm{m}^{2}{ }^{18}$

$H$. pylori serology testing was performed on the same day as the colonoscopy. Immunoglobulin G (IgG) antibody to $H$. pylori (Anti-H. pylori IgG Ab) were measured using IMMULITE $2000 \mathrm{H}$. pylori IgG produced by SIEMENS (Siemens Healthcare Diagnostics Products Ltd., Llanberis, UK) and a qualitative chemiluminescent immunometric assay. A subject was considered $H$. pylori infected when the $H$. pylori-IgG level was higher than $1.0 \mathrm{U} / \mathrm{mL}$.

\section{Assessment of endoscopic findings}

All EGD and colonoscopy were performed by board certi- fied gastrointestinal endoscopists. All the EGDs were performed with GIF-H260 (Olympus Optical Co., Ltd., Tokyo, Japan). All the colonoscopies were performed with CF-H260AI (Olympus Optical Co., Ltd.). During colonoscopy, the location, number, and size (estimated with opened biopsy forceps) of CRN were recorded. All CRN were biopsied or polypectomized and histologically evaluated. The histopathology of CRN was classified according to the World Health Organization classification. ${ }^{19}$ Advanced colorectal adenoma was defined as an adenoma that was $\geq 1 \mathrm{~cm}$ in diameter, had high-grade dysplasia, significant villous component or any combination thereof. Advanced CRN was defined as advanced adenoma and/or invasive cancer. The location of CRN was defined as distal colon (descending colon and rectosigmoid colon) and proximal colon (from cecum to splenic flexure).

Gastric mucosal atrophy was suggested when EGD findings showed whitish to yellowish mucosal color change, visible submucosal vessels, and/or absence of rugae. ${ }^{20}$ The extent of atrophy was classified into a closed type and an open type based on Kimura and Takemoto criteria. ${ }^{20}$ The closed type means that the atrophic border remains on the lesser curvature of the stomach, while the 0-type means that the atrophic border no longer exists on the lesser curvature but extends along the anterior and posterior walls of the stomach. ${ }^{20}$ In our study, AG was defined when the extent of atrophy showed the open type. Endoscopists recorded EGD findings and entered the diagnostic code into the electronic medical record system.

\section{Statistical analysis}

Clinical and endoscopic variables between groups are summarized as means and standard deviations for continuous variables or frequency (\%) for categorical variables. Associations of clinical and endoscopic variables with advanced CRN were tested using univariate logistic regression followed by multivariate analysis. Results are reported as odds ratios (ORs) and 95\% confidence interval (CI). All reported p-values were two-sided and p-values less than 0.05 were considered to indicate statistical significance. All statistical analyses were performed using SPSS $^{\circledR}$ Statistics version 21.0 (IBM Corp., Armonk, NY, USA).

\section{RESULTS}

\section{Baseline characteristics of the study population}

From January 2012 to November 2013, a consecutive series of 7,517 participants $\geq 40$ years of age who underwent screening colonoscopy were eligible for this study. A total of 6,351 subjects were included in the final analysis after exclusion of 1,166 participants because of a history of $H$. pylori eradication, incomplete colonoscopy, unavailable EGD data, and a history of colorectal cancer (Fig. 1).

The mean age of the 6,351 study patients was $51.7 \pm 8.1$ years and 3,491 subjects (54.9\%) were 50 years or older. Male com- 


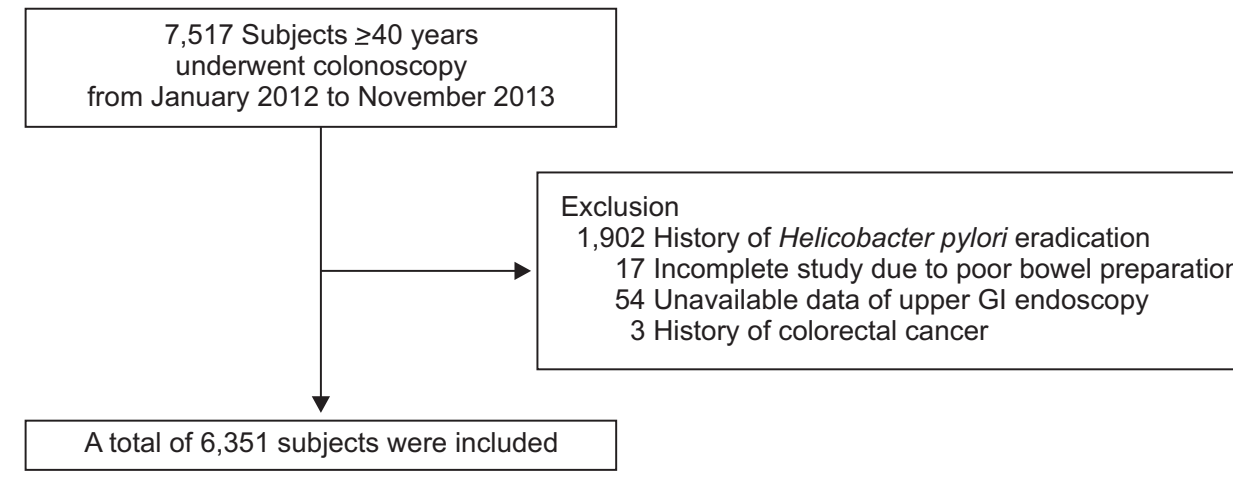

Fig. 1. Flow chart of the study population. GI, gastrointestinal.

Table 1. Baseline Characteristics of the Study Population

\begin{tabular}{|c|c|c|c|c|}
\hline Characteristic & All participants $(n=6,351)$ & H. pylori $(-)(\mathrm{n}=2,455)$ & H. pylori $(+)(\mathrm{n}=3,896)$ & p-value \\
\hline Male sex & $3,353(52.8)$ & $1,212(49.4)$ & $2,141(55.0)$ & $<0.001$ \\
\hline Age, yr & $51.7 \pm 8.1$ & $51.0 \pm 8.1$ & $52.1 \pm 8.1$ & $<0.001$ \\
\hline Family history of CRC & $291(4.6)$ & $98(4.0)$ & $193(5.0)$ & 0.074 \\
\hline Body mass index $\geq 25 \mathrm{~kg} / \mathrm{m}^{2}$ & $1,965(30.9)$ & 741 (30.2) & $1,224(31.4)$ & 0.300 \\
\hline Smoking habitus & & & & 0.011 \\
\hline Never & $3,551(55.9)$ & $1,430(58.2)$ & $2,121(54.4)$ & \\
\hline$<20$ pack year & $980(15.4)$ & $355(14.5)$ & $625(16.0)$ & \\
\hline$\geq 20$ pack year & $1,820(28.7)$ & $670(27.3)$ & $1,150(29.5)$ & \\
\hline Alcohol consumption & & & & 0.056 \\
\hline Never & $1,744(27.5)$ & $686(27.9)$ & $1,058(27.2)$ & \\
\hline$<20 \mathrm{~g} /$ day in women, $<40 \mathrm{~g} /$ day in men & $3,336(52.5)$ & $1,315(53.6)$ & $2,021(51.9)$ & \\
\hline$\geq 20 \mathrm{~g} /$ day in women, $\geq 40 \mathrm{~g} /$ day in men & $1,271(20.0)$ & $454(18.5)$ & $817(21.0)$ & \\
\hline Metabolic syndrome & $1,570(24.7)$ & $562(22.9)$ & $1,008(25.9)$ & 0.007 \\
\hline Waist circumferences, $\mathrm{cm}$ & $83.2 \pm 9.6$ & $82.5 \pm 9.7$ & $83.3 \pm 9.4$ & 0.005 \\
\hline Systolic blood pressure, $\mathrm{mm} \mathrm{Hg}$ & $122.9 \pm 15.8$ & $121.8 \pm 15.1$ & $123.6 \pm 16.2$ & $<0.001$ \\
\hline Diastolic blood pressure, $\mathrm{mm} \mathrm{Hg}$ & $77.9 \pm 11.7$ & $77.2 \pm 11.5$ & $78.4 \pm 11.9$ & $<0.001$ \\
\hline Fasting glucose, mg/dL & $98.9 \pm 22.3$ & $98.5 \pm 21.0$ & $99.1 \pm 23.0$ & 0.247 \\
\hline Triglyceride, mg/dL & $118.3 \pm 82.3$ & $117.3 \pm 79.4$ & $118.9 \pm 84.1$ & 0.447 \\
\hline HDL cholesterol, mg/dL & $56.0 \pm 14.7$ & $57.0 \pm 15.0$ & $55.4 \pm 14.4$ & $<0.001$ \\
\hline \multicolumn{5}{|l|}{ Colonoscopy findings } \\
\hline Overall CRN & 2,497 (39.2) & $864(35.2)$ & $1,633(41.9)$ & $<0.001$ \\
\hline Advanced CRN & $316(5.0)$ & $95(3.9)$ & $221(5.7)$ & 0.001 \\
\hline Villous adenoma & $45(0.7)$ & $13(0.5)$ & $32(0.8)$ & 0.177 \\
\hline Size $\geq 1 \mathrm{~cm}$ & $270(4.3)$ & $85(3.5)$ & $185(4.7)$ & 0.013 \\
\hline High-grade dysplasia or cancer & $51(0.8)$ & $11(0.4)$ & $40(1.0)$ & 0.012 \\
\hline No. of adenoma $\geq 3$ & $458(7.2)$ & $132(5.4)$ & $326(8.4)$ & $<0.001$ \\
\hline \multicolumn{5}{|l|}{ EGD findings } \\
\hline $\mathrm{AG}$ & $1,989(31.3)$ & $307(12.5)$ & $1,682(43.2)$ & $<0.001$ \\
\hline Gastric pathology & & & & 0.019 \\
\hline Gastric adenoma & $32(0.5)$ & $9(0.4)$ & $23(0.6)$ & \\
\hline High-grade dysplasia or cancer & $23(0.4)$ & $3(0.1)$ & $20(0.5)$ & \\
\hline
\end{tabular}

Data are presented as number (\%) or mean \pm SD. A chi-square test was used for categorical variables, and a t-test was used for continuous variables. H. pylori, Helicobacter pylori; CRC, colorectal cancer; HDL, high-density lipoprotein; CRN, colorectal neoplasm; EGD, esophagogastroduodenoscopy; AG, atrophic gastritis. 


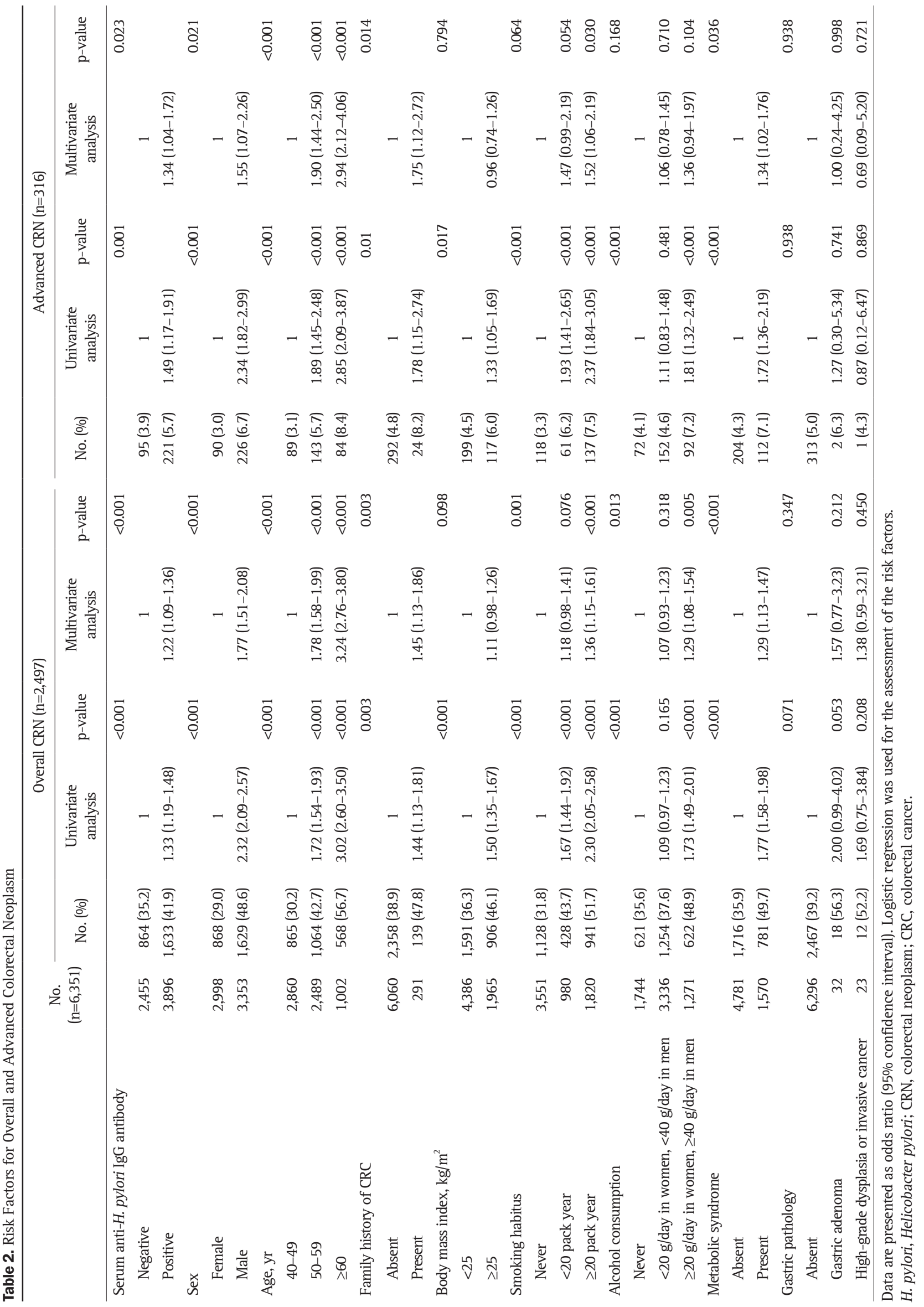


prised 52.8\% of the population. A total of 2,497 subjects (39.2\%) had CRN and 316 (5.0\%) had advanced CRN. AG was reported in 1,989 subjects (31.3\%). Detailed baseline characteristics are presented in Table 1.

\section{Association between $H$. pylori infection and CRN}

Of a total 6,351 subjects, the $H$. pylori-seropositivity was shown in $3,896(61.3 \%)$. The prevalence of overall CRN in subjects with $H$. pylori infection was significantly higher than those without $H$. pylori infection (41.9\% vs 35.2\%, p<0.001). The prevalence of advanced CRN in subjects with $H$. pylori infection was significantly higher than those without $H$. pylori infection (5.7\% vs 3.9\%, p=0.001) (Table 1). On the univariate analysis, male gender, older age, a family history of colorectal cancer, smoker, alcohol consumption, metabolic syndrome, BMI $\geq 25 \mathrm{~kg} / \mathrm{m}^{2}$ and $H$. pylori infection were significantly associated with overall and advanced CRN (Table 2). In logistic regression multivariate analysis, $H$. pylori infection was an independent risk factor of overall and advanced CRN. The OR of $H$. pylori infection for advanced CRN was 1.34 (95\% CI, 1.04 to 1.72; $\mathrm{p}=0.023$ ). Male gender, older age, a family history of colorectal cancer, smoking of $\geq 20$ pack year, and metabolic syndrome were also significantly associated with advanced CRN (Table 2).

\section{Association between $H$. pylori infection, AG, and advanced CRN}

A total of 6,351 subjects were classified according to the status of $H$. pylori infection and AG on EGD; 2,148 were $H$. pylori (-) AG (-), 307 were H. pylori (-) AG (+), 2,214 were H. pylori (+) AG (-), and 1,682 were H. pylori (+) AG (+). H. pylori (+) AG $(+)$ group showed significantly higher prevalence of overall CRN and advanced CRN (Table 3).

After logistic regression, H. pylori (+) AG (-) was associated with multiple adenomas (OR, 1.38; 95\% CI, 1.06 to 1.79; $\mathrm{p}=0.017)$ and proximal CRN (OR, 1.20; 95\% CI, 1.04 to 1.39; $\mathrm{p}=0.013$ ) (Table 3). H. pylori (-) AG (+) was associated with high-grade dysplasia, multiple adenomas and proximal CRN. H. pylori (+) AG (+) was significantly associated with advanced CRN, villous adenoma, $\geq 1 \mathrm{~cm}$ sized adenoma, high-grade dysplasia, multiple adenomas and proximal CRN.

After adjustment with confounding factors including age, gender, family history of colorectal cancer, BMI, metabolic syndrome, smoking status and amount of alcohol consumption, $H$. pylori (+) AG (+) was independently associated with advanced CRN (OR, 1.40; 95\% CI, 1.03 to $1.91 ; \mathrm{p}=0.030$ ) (Table 4). In addition, $H$. pylori (+) AG (+) was associated with high-grade dysplasia, multiple adenomas and proximal CRN. However, $H$. pylori (-) AG (+) was not an independent risk factor for any types of CRN. H. pylori (+) AG (-) also did not show any independent association with CRN except equivocal risk for overall CRN (OR, 1.13 ; 95\% CI, 0.99 to $1.29 ; \mathrm{p}=0.057$ ) and slightly increased risk of proximal CRN (OR, 1.17; 95\% CI, 1.01 to 1.36; p=0.036) (Table 4).

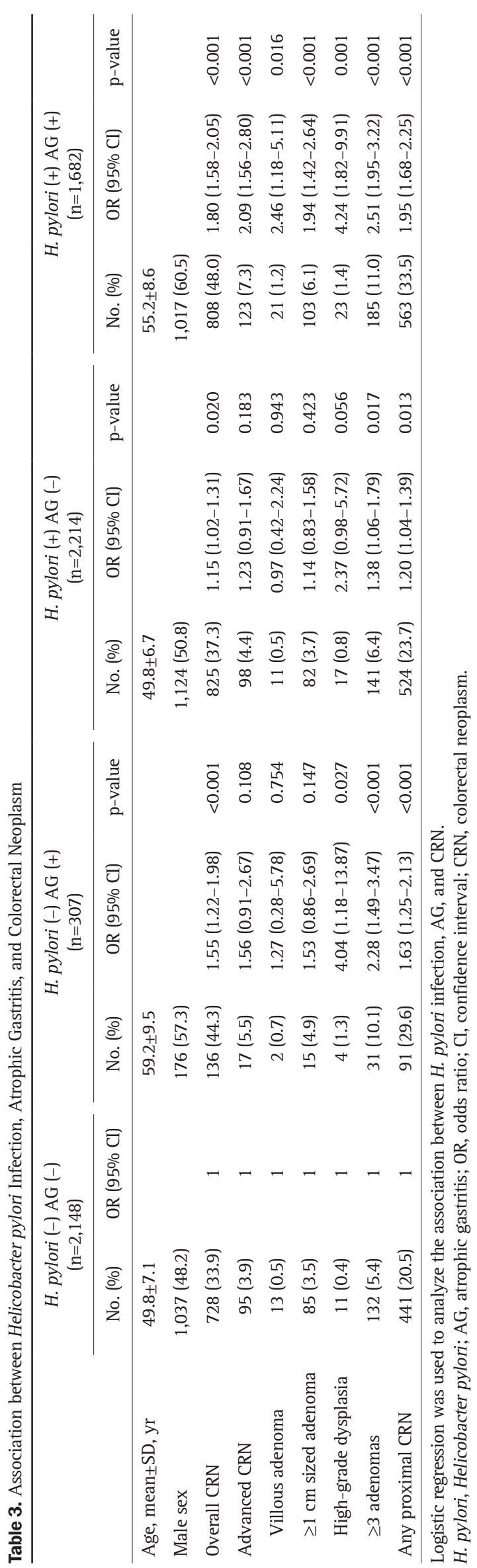


Table 4. Logistic Regression Model of the Association between Helicobacter pylori Infection, Atrophic Gastritis, and Colorectal Neoplasm after Adjustments for Confounding Factors*

\begin{tabular}{|c|c|c|c|c|c|c|c|}
\hline & \multirow{2}{*}{$\begin{array}{c}\text { H. pylori }(-) \text { AG (-) } \\
(\mathrm{n}=2,148) \\
\text { OR }(95 \% \mathrm{CI})\end{array}$} & \multicolumn{2}{|c|}{$\begin{array}{c}\text { H. pylori (-) AG (+) } \\
(\mathrm{n}=307)\end{array}$} & \multicolumn{2}{|c|}{$\begin{array}{c}\text { H. pylori (+) AG (-) } \\
(\mathrm{n}=2,214)\end{array}$} & \multicolumn{2}{|c|}{$\begin{array}{c}\text { H. pylori }(+) \text { AG (+) } \\
(\mathrm{n}=1,682)\end{array}$} \\
\hline & & OR $(95 \% \mathrm{CI})$ & $\mathrm{p}$-value & OR $(95 \% \mathrm{CI})$ & p-value & OR $(95 \% \mathrm{CI})$ & p-value \\
\hline Overall CRN & 1 & $0.85(0.65-1.11)$ & 0.222 & $1.13(0.99-1.29)$ & 0.057 & $1.24(1.07-1.42)$ & 0.003 \\
\hline Advanced CRN & 1 & $0.86(0.49-1.52)$ & 0.609 & $1.17(0.86-1.60)$ & 0.305 & $1.40(1.03-1.91)$ & 0.030 \\
\hline Villous adenoma & 1 & $1.01(0.21-4.79)$ & 0.989 & $0.93(0.40-2.16)$ & 0.871 & $2.00(0.93-4.30)$ & 0.076 \\
\hline$\geq 1 \mathrm{~cm}$ sized adenoma & 1 & $0.78(0.43-1.42)$ & 0.418 & $1.09(0.79-1.51)$ & 0.606 & $1.24(0.89-1.71)$ & 0.207 \\
\hline High-grade dysplasia & 1 & $2.40(0.66-8.71)$ & 0.180 & $2.22(0.92-5.38)$ & 0.077 & $2.91(1.22-6.98)$ & 0.017 \\
\hline$\geq 3$ adenomas & 1 & $0.88(0.55-1.39)$ & 0.588 & $1.29(0.98-1.69)$ & 0.069 & $1.39(1.06-1.83)$ & 0.017 \\
\hline Any proximal CRN & 1 & $0.82(0.61-1.09)$ & 0.182 & $1.17(1.01-1.36)$ & 0.036 & $1.29(1.10-1.51)$ & 0.002 \\
\hline
\end{tabular}

Logistic regression was used to analyze the data.

H. pylori, Helicobacter pylori; AG, atrophic gastritis; OR, odds ratio; CI, confidence interval; CRN, colorectal neoplasm.

*Adjustments were performed by incorporating all of the relevant factors, such as age, gender, family history of colorectal cancer, body mass index, metabolic syndrome, pack-years of smoking, and level of alcohol consumption, into the analysis.

\section{DISCUSSION}

Our cross-sectional study demonstrated that $H$. pylori infection was an independent risk factor of advanced CRN. We found that this increased risk of advanced CRN by $H$. pylori infection was evident only in the presence of AG.

The association between $H$. pylori infection and CRN has been inconclusive. Several meta-analyses and large population based studies showed the positive association between $H$. pylori and CRN. ${ }^{6-8,21,22}$ However, other studies did not find any relationship between $H$. pylori and CRN or showed limited associations only in women. ${ }^{10,11}$ The reason for this inconsistency between studies is not clear. Many diagnostic tests for $H$. pylori such as serological test, histological examination, the urea breath test and the rapid urease test are currently available. Difference in methods of $H$. pylori detection between studies might contribute to the inconsistent results about the relation between $H$. pylori and CRN. Another reason for inconsistent findings between studies may be related to different study participants and small sample sizes. Unlike absence of association between $H$. pylori and CRN in previous small sized, hospital based studies, ${ }^{10,11}$ a recent large scale $(\mathrm{n}=2,195)$ cross-sectional study using $H$. pylori IgG serology showed 2.21-fold higher prevalence of advanced CRN in the H. pylori (+) group. ${ }^{6}$ A large population based case-control study which enrolled 1,669 controls and 1,712 patients with colorectal cancer also showed the positive association between $H$. pylori and colorectal cancer. ${ }^{7}$ In our large cohort including 6,351 subjects, we found $H$. pylori infection was a significant, independent risk factor for advanced CRN with OR 1.34 (Table 2). We suggest that large study population should be necessary in this kind of study, especially in Asia, because high prevalence of $H$. pylori colonization in Eastern countries may conceal an association between $H$. pylori and CRN if the association is modest.
Our study showed that advanced CRN occurred more frequently in $H$. pylori infected patients with AG than without AG (7.3\% vs 4.4\%, $\mathrm{p}<0.001)$. After adjustment with confounders, H. pylori (+) AG (+) was still independently associated with advanced CRN, but $H$. pylori (+) AG (-) was not. This finding is consistent with another recent study which showed that the $H$. pylori-positive gastritis and intestinal metaplasia, a more easily recognizable form of mucosal atrophy, increased risk for CRN while $H$. pylori-negative gastritis did not. ${ }^{16}$ Although other studies did not show the relation between AG and CRN, these negative studies excluded those with $H$. pylori infection or did not analyze $H$. pylori infection status. ${ }^{23}$ Thus we believe that AG accompanied by $H$. pylori infection may be positively associated with advanced CRN. In addition, when considering $H$. pylori (+) AG (-) did not increase the risk of advanced CRN, long-standing $H$. pylori infection may be crucial for development of advanced CRN because AG is usually a chronic sequela of $H$. pylori infection. In fact, our data also showed mean age was older in $H$. pylori (+) AG (+) group than in H. pylori (+) AG (-) group (55.2 years vs 49.8 years), which suggests $H$. pylori (+) AG (+) group may have suffered from longer period of $H$. pylori infection because the acquisition of $H$. pylori in Korean population is presumed to be early in their lives and almost similar throughout the population. ${ }^{24}$ Persistent exposure to $H$. pylori infection directly increased gastrin secretion. ${ }^{25}$ Moreover, persistent exposure to $H$. pylori induced atrophic changes of the gastric body mucosa, which resulted in increased level of serum gastrin by feedback of low acid output on the antral G cell. ${ }^{26}$ Gastrin has a trophic effect on epithelial cell growth and proliferation, which can contribute to colorectal carcinogenesis. ${ }^{27}$ Others speculated that hypochlorhydria caused by $H$. pylori-related AG might lead to bacterial overgrowth in the gastrointestinal tract and alterations in the colonic microenvironment of the bacterial flora. ${ }^{28}$ Additionally, hypochlorhydria may result in an increase in un- 
absorbed nutrients in the lower intestine due to impaired gastric protein digestion. ${ }^{29}$ Therefore, some metabolites such as hydrogen sulfide, ammonia or phenol which can derive from fermentation of malabsorbed proteins by overgrown bacteria probably play a role in the pathogenesis of CRN.

In our study, H. pylori (-) AG (+) was not an independent risk factor for any types of CRN. Some portion of patients with $H$. pylori (-) AG (+) may represent a group of severe AG following long-term $H$. pylori infection. Thus, this group may show hypergastrinemia and, theoretically, higher risk of CRN. Despite this hypothetical reasoning, our study did not show any correlation. We suggest that the relatively small number $(n=307)$ and older age of the $H$. pylori (-) AG (+) group might mask the probable influence on CRN in the multivariate analysis. When we performed a multivariate analysis after exclusion of age factor, $H$. pylori (-) AG (+) group was an independent risk factor for CRN with high-grade dysplasia (data not shown).

In this study, $H$. pylori infection, especially with the presence of AG, increased the risk of proximal CRN. This finding is consistent with a previous study. ${ }^{8}$ The risk of CRN associated with proximal and distal CRN are different in environmental risk factors and genetic alterations. ${ }^{30} \mathrm{CpG}$ island methylator phenotype and microsatellite instability are shown predominantly in the proximal CRN and altered DNA methylation is more frequently involved in the carcinogenic process of the proximal colon. ${ }^{31}$ We suggest AG induced colonic bacterial overgrowth may generate methylation changes more easily in the proximal colon. In addition, colonic bacterial overgrowth leads to an enhanced production of secondary bile acids which can increase the risk for proximal colon cancer. ${ }^{32}$ This finding may further explain our results of increased risk of proximal CRN in those with $H$. pylori-related AG.

Our study had several limitations. First, we diagnosed AG endoscopically based on Kimura and Takemoto criteria ${ }^{20}$ without histologic diagnosis, which is not completely objective. Therefore, there may be interobserver variability regarding the diagnosis of AG. ${ }^{33}$ Although we could not investigate the interobserver variability because of the retrospective study design, we suggest the variability might not be high because all the endoscopists who performed the study EGD and colonoscopies were board-certified gastrointestinal endoscopists. Second, H. pylori infection was assessed by the serology test, which cannot accurately reflect the current $H$. pylori infection. Third, serum gastrin level was not obtained which could explain key mechanisms how $H$. pylori affect the development of CRN. Finally, this study was a single center investigation which requires further studies in other nations and ethnic groups before generalization of the conclusion. Despite these limitations, our study has strength that we analyzed the relation not only between $H$. pylori infection and CRN but also further investigated the role of AG.

In conclusion, this study indicated that $H$. pylori infection increased the risk of overall and advanced CRN. When $H$. pylori infection status was stratified by the presence of AG, only $H$. pylori (+) AG (+) group enhanced the risk of advanced CRN. Strict colonoscopy screening and surveillance may be warranted in those with endoscopic AG and H. pylori infection.

\section{CONFLICTS OF INTEREST}

No potential conflict of interest relevant to this article was reported.

\section{REFERENCES}

1. Schistosomes, liver flukes and Helicobacter pylori. IARC Working Group on the evaluation of carcinogenic risks to humans. Lyon, 7-14 June 1994. IARC Monogr Eval Carcinog Risks Hum 1994;61:1-241

2. Parkin DM. The global health burden of infection-associated cancers in the year 2002. Int J Cancer 2006;118:3030-3044.

3. Roubaud Baudron C, Franceschi F, Salles N, Gasbarrini A. Extragastric diseases and Helicobacter pylori. Helicobacter 2013;18 Suppl 1:44-51.

4. Selgrad M, Bornschein J, Rokkas T, Malfertheiner P. Helicobacter pylori: gastric cancer and extragastric intestinal malignancies. Helicobacter 2012;17 Suppl 1:30-35.

5. Meucci G, Tatarella M, Vecchi M, et al. High prevalence of Helicobacter pylori infection in patients with colonic adenomas and carcinomas. J Clin Gastroenterol 1997;25:605-607.

6. Hong SN, Lee SM, Kim JH, et al. Helicobacter pylori infection increases the risk of colorectal adenomas: cross-sectional study and meta-analysis. Dig Dis Sci 2012;57:2184-2194.

7. Zhang Y, Hoffmeister M, Weck MN, Chang-Claude J, Brenner H. Helicobacter pylori infection and colorectal cancer risk: evidence from a large population-based case-control study in Germany. Am J Epidemiol 2012;175:441-450.

8. Inoue I, Mukoubayashi C, Yoshimura N, et al. Elevated risk of colorectal adenoma with Helicobacter pylori-related chronic gastritis: a population-based case-control study. Int J Cancer 2011;129:2704-2711

9. Fujimori S, Kishida T, Kobayashi T, et al. Helicobacter pylori infection increases the risk of colorectal adenoma and adenocarcinoma, especially in women. J Gastroenterol 2005;40:887-893.

10. Siddheshwar RK, Muhammad KB, Gray JC, Kelly SB. Seroprevalence of Helicobacter pylori in patients with colorectal polyps and colorectal carcinoma. Am J Gastroenterol 2001;96:84-88.

11. Machida-Montani A, Sasazuki S, Inoue M, et al. Atrophic gastritis, Helicobacter pylori, and colorectal cancer risk: a case-control study. Helicobacter 2007;12:328-332.

12. Adamu MA, Weck MN, Gao L, Brenner H. Incidence of chronic atrophic gastritis: systematic review and meta-analysis of followup studies. Eur J Epidemiol 2010;25:439-448.

13. Kuipers EJ, Uyterlinde AM, Peña AS, et al. Long-term sequelae of Helicobacter pylori gastritis. Lancet 1995;345:1525-1528. 
14. de Vries AC, van Grieken NC, Looman CW, et al. Gastric cancer risk in patients with premalignant gastric lesions: a nationwide cohort study in the Netherlands. Gastroenterology 2008;134:945952.

15. Yang MH, Son HJ, Lee JH, et al. Do we need colonoscopy in patients with gastric adenomas? The risk of colorectal adenoma in patients with gastric adenomas. Gastrointest Endosc 2010;71:774781.

16. Sonnenberg A, Genta RM. Helicobacter pylori is a risk factor for colonic neoplasms. Am J Gastroenterol 2013;108:208-215.

17. Grundy SM, Cleeman JI, Daniels SR, et al. Diagnosis and management of the metabolic syndrome: an American Heart Association/ National Heart, Lung, and Blood Institute Scientific Statement. Circulation 2005;112:2735-2752.

18. World Health Organization, Regional Office for the Western Pacific. The Asia-Pacific perspective: redefining obesity and its treatment. Sydney: Health Communications Australia, 2000.

19. Bosman FT, Carneiro F, Hruban RH, Theise ND. WHO classification of tumours of the digestive system. 4th ed. Lyon: WHO Press, 2010.

20. Kimura K, Takemoto T. An endoscopic recognition of the atrophic border and its significance in chronic gastritis. Endoscopy 1969;1:87-97.

21. Zhao YS, Wang F, Chang D, Han B, You DY. Meta-analysis of different test indicators: Helicobacter pylori infection and the risk of colorectal cancer. Int J Colorectal Dis 2008;23:875-882.

22. Wu Q, Yang ZP, Xu P, Gao LC, Fan DM. Association between Helicobacter pylori infection and the risk of colorectal neoplasia: a systematic review and meta-analysis. Colorectal Dis 2013;15:e352e364.

23. Lahner E, Sbrozzi-Vanni A, Vannella L, et al. No higher risk for colorectal cancer in atrophic gastritis-related hypergastrinemia.
Dig Liver Dis 2012;44:793-797.

24. Jung JH, Choi KD, Han S, et al. Seroconversion rates of Helicobacter pylori infection in Korean adults. Helicobacter 2013;18:299308.

25. Ciccotosto GD, McLeish A, Hardy KJ, Shulkes A. Expression, processing, and secretion of gastrin in patients with colorectal carcinoma. Gastroenterology 1995;109:1142-1153.

26. Watson SA, Grabowska AM, El-Zaatari M, Takhar A. Gastrin: active participant or bystander in gastric carcinogenesis? Nat Rev Cancer 2006;6:936-946.

27. Thorburn CM, Friedman GD, Dickinson CJ, Vogelman JH, Orentreich N, Parsonnet J. Gastrin and colorectal cancer: a prospective study. Gastroenterology 1998;115:275-280.

28. Kanno T, Matsuki T, Oka M, et al. Gastric acid reduction leads to an alteration in lower intestinal microflora. Biochem Biophys Res Commun 2009;381:666-670.

29. Evenepoel P, Claus D, Geypens B, et al. Evidence for impaired assimilation and increased colonic fermentation of protein, related to gastric acid suppression therapy. Aliment Pharmacol Ther 1998;12:1011-1019.

30. Iacopetta B. Are there two sides to colorectal cancer? Int J Cancer 2002;101:403-408.

31. Nakajima T, Enomoto S, Ushijima T. DNA methylation: a marker for carcinogen exposure and cancer risk. Environ Health Prev Med 2008;13:8-15.

32. Giovannucci E, Colditz GA, Stampfer MJ. A meta-analysis of cholecystectomy and risk of colorectal cancer. Gastroenterology 1993;105:130-141.

33. Laine L, Cohen H, Sloane R, Marin-Sorensen M, Weinstein WM. Interobserver agreement and predictive value of endoscopic findings for H. pylori and gastritis in normal volunteers. Gastrointest Endosc 1995;42:420-423. 